\title{
Toxic Megacolon - A Three Case Presentation
}

\author{
Irina Magdalena Dumitru, Eugen Dumitru², Sorin Rugina ${ }^{1,3}$, Liliana Ana Tuta $^{2}$ \\ ${ }^{1}$ Discipline of Infectious Diseases, Faculty of Medicine, Ovidius University of Constanta, Romania \\ ${ }^{2}$ Discipline of Internal Medicine, Faculty of Medicine, Ovidius University of Constanta, Romania \\ ${ }^{3}$ The Academy of Romanian Scientists
}

\begin{abstract}
Introduction: Toxic megacolon is a life-threatening disease and is one of the most serious complications of Clostridium difficile infection (CDI), usually needing prompt surgical intervention. Early diagnosis and adequate medical treatment are mandatory.

Cases presentation: In the last two years, three Caucasian female patients have been diagnosed with toxic megacolon and treated in the Clinical Infectious Diseases Hospital, Constanta. All patients had been hospitalized for nonrelated conditions. The first patient was in chemotherapy for non-Hodgkin's lymphoma, the second patient had undergone surgery for colon cancer, and the third patient had surgery for disc herniation. In all cases the toxin test $(A+B)$ was positive and ribotype 027 was present. Abdominal CT examination, both native and after intravenous contrast, showed significant colon dilation, with marked thickening of the wall. Resolution of the condition did not occur using the standard treatment of metronidazole and oral vancomycin, therefore the therapy was altered in two cases using intracolonic administration of vancomycin and intravenous tigecycline.

Conclusions: In these three cases of $\mathrm{CDI}$, the risk factors for severe evolution were: concurrent malignancy, renal failure, obesity, and immune deficiencies. Ribotype 027, a marker for a virulent strain of CD, was found in all three cases complicated by toxic megacolon. The intracolonic administration of vancomycin, and intravenous tigecycline was successful when prior standard therapy had failed, and surgery was avoided.
\end{abstract}

Keywords: toxic megacolon, Clostridium difficile, treatment

Received: 6 January 2017 / Accepted: 30 January 2017

\section{INTRODUCTION}

Toxic megacolon is a rare but life-threatening disease and may be defined as the severe dilatation of the colon associated with colitis. The main causes are inflammatory bowel diseases (IBDs) such as ulcerative colitis and Crohn's disease. Toxic megacolon is one of the most serious complication of Clostridium difficile infection (CDI) [1].

The risk factors for CDI are: advanced age (persons $>65$ years), inflammatory bowel diseases, immunodeficiency (human immunodeficiency virus infection, hematologic malignancies, chronic kidney disease) increased duration of hospitalization, exposure to antibiotics or chemotherapeutic agents, the use of proton pump inhibitors and upper gastro-intestinal tract surgery [2].

It has been shown that fulminant evolution of CDI results from toxin-induced proinflammatory cytokine released into the colon, followed by increased vascular permeability and host cell necrosis [3] and is associ- ated with the hypervirulent 027 strain of Clostridium difficile [4].

Certain risk factors may precipitate the progression of CDI to toxic megacolon and include long-term antibiotic therapy, concurrent malignancy, chronic obstructive pulmonary disease, renal failure, immunosuppressive medication [5], exposure to prokinetic agents [6] or proton pump inhibitors $[7,8]$. In critically ill patients, the severity of underlying disease, surgery procedures, length of hospitalization, multiple admissions, and one or more organs dysfunction, are also factors predisposing to a high risk of CDI [8].

The incidence of toxic megacolon in patients with colitis induced by Clostridium difficile is low and ranges between $0.4 \%$ and $3 \%$ [9]. However, the mortality rate is very high, and varies from $38 \%$ to $80 \%$ [10]. The absence of diarrhea due to ileus, rapid abdominal distension, abdominal pain and tenderness, tachycardia and hypotension in patients diagnosed with CDI are important signs of a progression to toxic megacolon [9]. The diagnosis of toxic megacolon needs radiological 
proof of colonic dilatation in excess of $6 \mathrm{~cm}$. CT scan findings, such as colonic wall thickening, distortion of haustral folds, and ascites are helpful in confirming the diagnosis of a toxic megacolon [10].

The treatment of toxic megacolon in CDI remain a challenge. Drug therapy remains controversial due to the frequently resultant poor results. In such cases, surgical treatment must be considered as an alternative approach [11].

Due to the increased mortality, which occurs in cases associated with colonic perforation, peritonitis, septic shock and multiple organ dysfunction, early diagnosis and treatment are crucial [2].

Three cases where the diagnosis was toxic megacolon caused by the CDI, and which were successfully treated by drug therapy alone, are presented.

\section{CASES PRESENTATION}

The three cases presented below were Caucasian female patients with severe CDI hospitalized in the Clinical Infectious Diseases Hospital, Constanta, between $\mathrm{Au}-$ gust 2014 and May 2016.

They had prolonged hospitalizations before admission to the department of infectious diseases. The first patient had spent three weeks in the hematology department, where she received chemotherapy for a non-Hodgkin's lymphoma and ten days of antibiotic treatment with ceftriaxone $1 \mathrm{~g}$ IV q12hrs, and ciprofloxacin $400 \mathrm{mg}$ IV q12hrs, for an acute bacterial pneumonia. After discharge from hospital, diarrheal stools occurred and the patient received ambulatory symptomatic treatment for two weeks without any improvement of symptoms.
The second patient was hospitalized for three weeks in the general surgery clinic, where she underwent surgery intervention for colon cancer. During hospitalization, she was diagnosed with a urinary tract infection with $E$. coli, for which she received a seven-day treatment with oral ciprofloxacin 500 mg PO q12hrs. Diarrheal stools occurred subsequently, at which point only symptomatic treatment was provided.

The third patient had surgery for a herniated disc. Two interventions were undertaken within three months in the neurosurgery clinic. During this time, the patient had a urinary catheter placed and had repeated urinary tract infections. Ciprofloxacin $500 \mathrm{mg}$ PO q12hrs was prescribed, and continued for more than two weeks.

None of the patients were known to have with chronic hepatitis B, HIV, tuberculosis or inflammatory bowel disease, or had received treatment with proton pump inhibitors in the last six months. Only one patient recently had been diagnosed with chronic viral $\mathrm{C}$ hepatitis, with a F3 fibrosis score. Another had been diagnosed with rheumatoid arthritis, but had not taken any specific medication for this within the last two years, except short courses of nonsteroidal anti-inflammatory drugs (seven-day courses of either meloxicam PO 15 $\mathrm{mg}$ /day, or ibuprofen $400 \mathrm{mg}$ PO q12hrs).

None of our patients was consuming alcohol or drugs, and all had a BMI greater than $30 \mathrm{~kg} / \mathrm{m}^{2}$ (Table 1).

On admission, all patients were hemodynamically stable, presenting with a temperature in excess of $38.5^{\circ}$ $\mathrm{C}$, signs of dehydration, nausea, abdominal cramps, oliguria, peripheral edema, ascites. In two patients, there was evidence of bilateral pleural effusion (Table 2).

Table 1. Patients' characteristics - demographics and diseases history

\begin{tabular}{lccc} 
& Case $\mathbf{1}$ & Case 2 & Case 3 \\
Age (years) & 67 & 75 & 59 \\
Body mass index (BMI) $\left(\mathrm{kg} / \mathrm{m}^{2}\right)$ & 30.5 & 30.1 & 32.3 \\
Environmental origin & Urban & Rural & Urban \\
Chronic hepatitis B/C & No/YES & No/No & No/No \\
HIV/AIDS & No & No & YES \\
Autoimmune diseases & No & No & No \\
Inflammatory bowel disease (IBD) & No & No & No \\
Treatment with proton pump inhibitors & No & No & No \\
Alcohol consumption & No & No & No \\
Drug users & No & Colon cancer & Neurosurgical intervention \\
Pathological recent history & Non-Hodgkin lymphoma & surgery & for a herniated disc \\
Antibiotic treatment & chemotherapy & ciprofloxacin & ciprofloxacin \\
\hline
\end{tabular}


Table 2. Clinical and laboratory patients' characteristics

\begin{tabular}{|c|c|c|c|}
\hline & Case 1 & Case 2 & Case 3 \\
\hline Fever (max) & $39.0^{\circ} \mathrm{C}$ & $38.8^{\circ} \mathrm{C}$ & $39.2^{\circ} \mathrm{C}$ \\
\hline Heart rate $>90 /$ minute, & yes & yes & yes \\
\hline Respiratory rate $>20 /$ minute & yes & yes & yes \\
\hline Peripheral edema & yes & yes & yes \\
\hline Ascites & yes & yes & yes \\
\hline Pleural effusion & yes & no & yes \\
\hline Low diuresis & yes & yes & yes \\
\hline Acute renal failure & yes & yes & yes \\
\hline White cell count $\left(10^{9} / \mathrm{L}\right)$ & $23.0 \times 10^{9} / \mathrm{L}$ & $25.0 \times 10^{9} / \mathrm{L}$ & $30.0 \times 10^{9} / \mathrm{L}$ \\
\hline C-reactive protein (mg/dL) & 16.8 & 18.6 & 15.9 \\
\hline Blood urea nitrogen (mg/dL) & 102 & 89 & 98 \\
\hline Serum albumin levels $<2.5 \mathrm{~g} / \mathrm{dL}$ & yes & yes & yes \\
\hline ATLAS score & 6 & 7 & 6 \\
\hline Toxin test $(A+B)$ & positive & positive & positive \\
\hline Ribotype 027 & yes & yes & yes \\
\hline
\end{tabular}

Laboratory tests showed a marked leukocytosis $\left(20.0 \times 10^{9} / \mathrm{L}\right)$ with neutrophilia, C-reactive protein greatly increased (>15 mg/dl), hypoalbuminemia and signs of acute renal failure associated with oliguria, increased blood urea nitrogen and serum creatinine.

In all cases the toxin test $(\mathrm{A}+\mathrm{B})$ was positive, and ribotype 027 was present (Table 2).

The ATLAS score was calculated which includes age, treatment with systemic antibiotics, leucocyte count, albumin, and serum creatinine. A white blood cell count in excess of $15 \times 10^{9} / \mathrm{L}$, rising serum creatinine, temperature greater than $38.5^{\circ} \mathrm{C}$ and levels of serum albumin less than $2.5 \mathrm{mg} / \mathrm{dL}$ were considered predic- tors of severe CDI.

For this reason, the patients received initial treatment with intravenous metronidazole, $1.5 \mathrm{~g} /$ day divided into 3 doses, and oral vancomycin, $125 \mathrm{mg}$, four times daily as well as parenteral hydration and albumin infusions. After a period of 48-72 hours all three presented with increased temperature $\left(>39.0^{\circ} \mathrm{C}\right)$, increased abdominal cramping, significant bloating of the abdomen and vomiting. Laboratory tests revealed marked leukocytosis $\left(29.0 \times 10^{9} / \mathrm{L}, 32.0 \times 10^{9} / \mathrm{L}\right.$ and $38.0 \times 10^{9} / \mathrm{L}$, respectively).

Abdominal CT examination, both without contrast medium (Figure 1A and B) and with intravenous con-
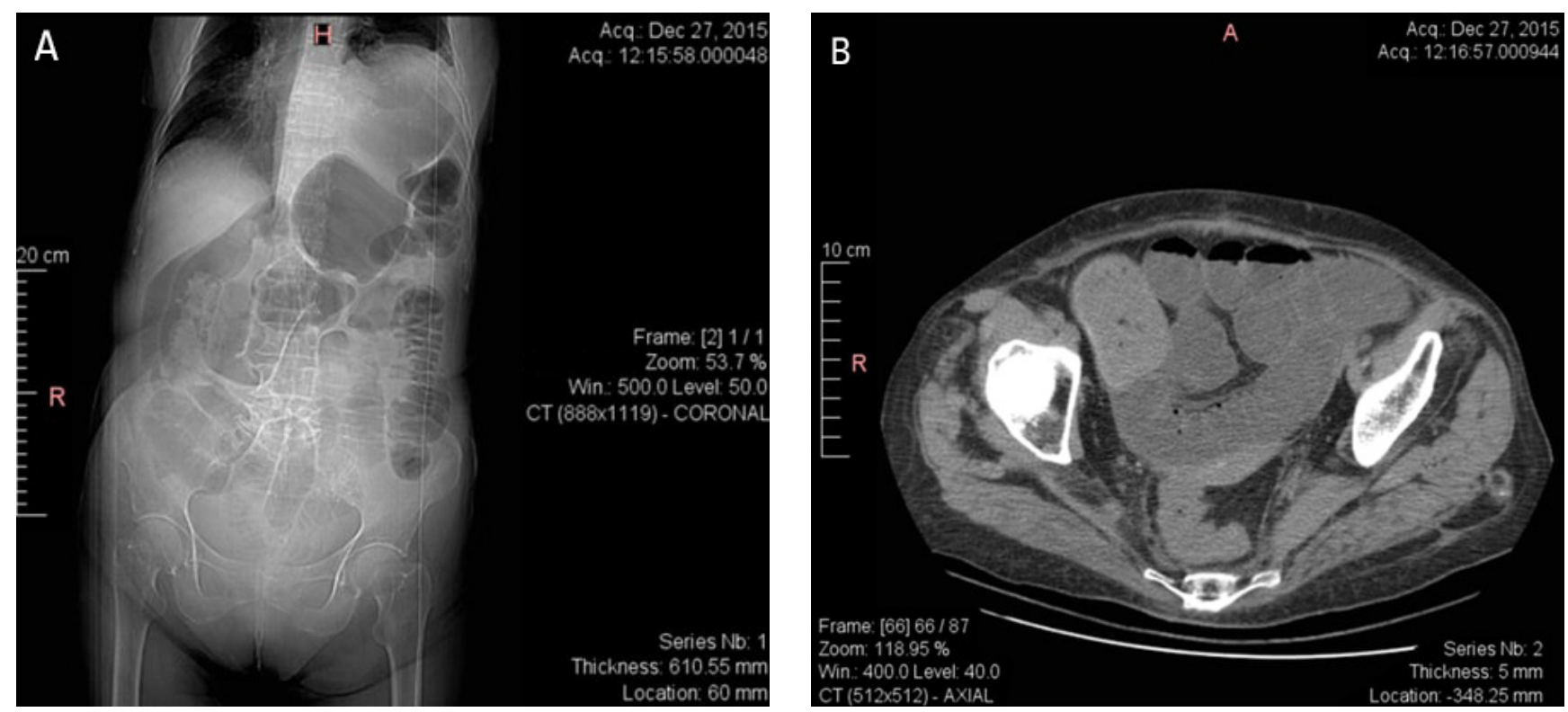

Fig.1A and B. Colon significantly dilated, with marked thickening of the wall (without contrast medium) 

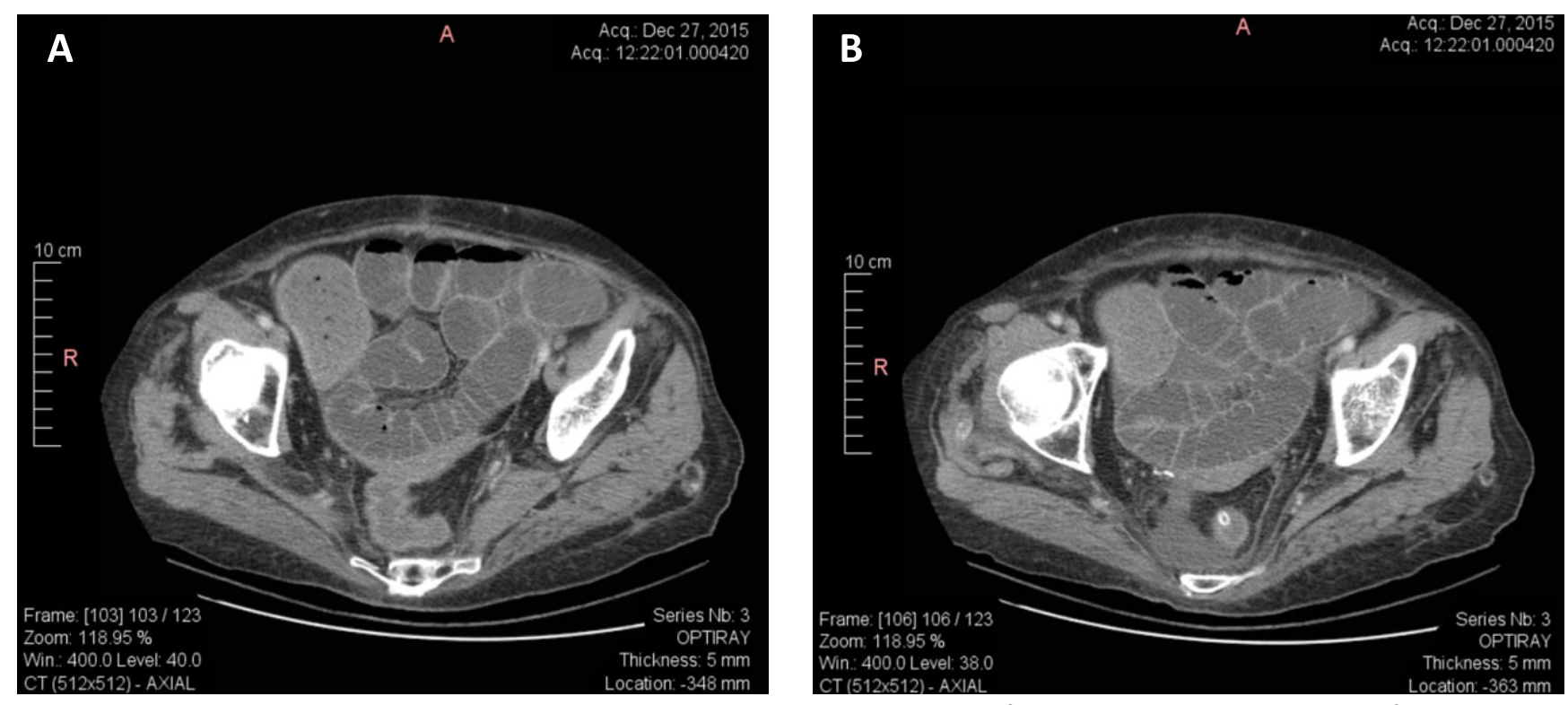

Fig. 2A and B. Colon significantly dilated, with marked thickening of the wall (intravenous contrast medium)

trast medium (Figure $2 \mathrm{~A}$ and $\mathrm{B}$ ), showed that the colon was significantly dilated, with marked thickening of the wall. This suggested a diagnosis of toxic megacolon.

The APACHE II scores for each patient were $26 \mathrm{pts}$ in the first case, 29 pts in the second and 24 pts in the third, with an estimated mortality of $56.9 \%, 67.2 \%$ and respectively $49.7 \%$.

Because severe CDI was considered possible, tigecycline therapy was started, $100 \mathrm{mg}$ IV infusion, then 50 mg IV infusion q12hrs. In addition, intravenous metronidazole was administrated, $1.5 \mathrm{~g} /$ day divided into 3 doses and oral vancomycin, $1 \mathrm{~g}$ /day, divided into 4 doses. Supportive measures, including intravenous fluid resuscitation and electrolyte replacement were given to all patients and a nasogastric tube (NGT) was placed to assist with gastrointestinal decompression.

In two patients, there was a rapid improvement in their condition. After three days, there was an absence of fever with an improvement in their clinical and paraclinical status, with declined leukocytosis $\left(16.0 \times 10^{9} / \mathrm{L}\right.$ and $\left.19.0 \times 10^{9} / \mathrm{L}\right)$ and C-reactive protein $(8.2 \mathrm{mg} / \mathrm{dL}$ and $8.9 \mathrm{mg} / \mathrm{dL}$ ).

In the third case, the general condition was worsened. Her temperature rose to over $39.5^{\circ} \mathrm{C}$, and she had considerable abdominal pain, pronounced abdominal distention and vomiting. The APACHE II score increased to 26 pts. For this reason, tigecycline was removed from the regimen, and the vancomycin dose was increased to $2 \mathrm{~g}$ /day orally, administered by nasogastric tube, divided into 4 doses plus vancomycin,
$1 \mathrm{~g} /$ day in enema. After other two days, the patient's general condition improved.

All three patients received human albumin, depending on the amount of losses and hydro- electrolytic balance.

After ten days of treatment all patients improved. Abdominal distension disappeared, there was no ede$\mathrm{ma}$ and ascites and feces were of normal appearance. The complete blood count $(C B C)$ was within normal limits (leukocytes $8.9 \times 10^{9} / \mathrm{L}, 9.1 \times 10^{9} / \mathrm{L}$ and respectively $8.0 \times 10^{9} / \mathrm{L}$ ), inflammatory markers were absent, $\mathrm{C}$-reactive protein was less than $1 \mathrm{mg} / \mathrm{dL}$. The erythrocyte sedimentation rate was $12 \mathrm{~mm} / \mathrm{h}$ and serum albumin was normal.

The hospitalization periods were between 14 to 21 days, and all three patients were discharged having good general condition. They were followed up for 6 months after hospital discharge, and no relapses were recorded.

\section{DIscussions}

In the last four years, 220 cases with Clostridium difficile diarrhea were reported, but only three cases of toxic megacolon were registered ( $1.36 \%$ incidence).

Numerous studies have shown that there are certain risk factors favoring the evolution from mild diarrhea to toxic megacolon in patients with CDI including older age and underlying co-morbidities, significant leukocytosis, hypoalbuminemia and acute renal failure $[10,11,12]$. In our case, two of the three patients were 
older than 65 years, and all three had all the other risk factors listed above. Hypoalbuminemia (serum albumin levels $<2.0 \mathrm{~g} / \mathrm{dL}$ ) is caused by the inflammation of the bowel wall that allows leakage of albumin into the lumen, associated with an inadequate compensatory liver synthesis. Ascites and peripheral edema may be observed in these situations $[11,12]$.

Obesity was also considered as a risk factor in CDI. In a cohort of 196 patients with CDI hospitalized in a gastroenterology department, Mulki (2016) identify a statistically significant association between BMI and severe infection with Clostridium difficile. The authors' conclusion was that $\mathrm{BMI}>35 \mathrm{~kg} / \mathrm{m}^{2}$ was more frequently associated with severe CDI, compared to a BMI 20$35 \mathrm{~kg} / \mathrm{m}^{2}[13]$.

Regarding the relationship between chronic viral hepatitis B or C and CDI, it is reported that there is an alteration of the gut microbiota in liver cirrhosis and thus a higher risk of CDI. But, it has not been demonstrated that chronic viral hepatitis B or C, regardless of the stage of fibrosis, is a risk factor for a severe form of CDI $[14,15]$. The same thing can be said about CDI association with autoimmune diseases. It is known that there is a causal relationship between the presence of rheumatoid arthritis and CDI, rather between methotrexate therapy and CDI. Naimushin (2011) presents a case of CDI in a patient with rheumatoid arthritis under methotrexate therapy, in whom Clostridium difficile-associated colitis had developed without prior antibiotic treatment $[16,17]$. Moreover, a change in gut microbiota in patients with rheumatoid arthritis could favor the occurrence of CDI [18].

In all three patients, the epidemic strain ribotype 027 was detected which is known to be associated with an increased severity of disease and high mortality [12, $19,20]$. However, the detection of toxin A and/or B in feces by EIA has not been established as a predictor of CDI severity [21].

Malignancies and chemotherapy are frequently associated with severe forms of CDI. Duberkke (2007) found that $57 \%$ patients who had various forms of cancer and chemotherapy developed severe CDI [22,23].

Regarding therapy for patients with severe CDI who are unresponsive to metronidazole and vancomycin, several studies have reported good results after intracolonic administration of vancomycin. Akamine (2016), in a study of 696 patients with severe CDI, demonstrated that the intracolonic administration of vancomycin therapy was beneficial in unresponsive CDI patients
[24]. In our patients, especially in patient number three, intracolonic vancomycin treatment improved the overall condition and helped in the remission of toxic megacolon.

Tigecycline, a new glycylcycline, has been shown to have favorable activity against Clostridium difficile and is now recommended for the treatment of CDI, including severe cases refractory to other treatments, in combination with metronidazole and vancomycin $[25,26]$. In our study, only two of the three patients progressed satisfactorily after administration of tigecycline. Both Larson KC (2011) and Britt (2014) reported an 85.7\% clinical cure after therapy with metronidazole, oral vancomycin and intravenous tigecycline [27].

\section{- CONCLUSIONS}

Toxic megacolon occurred as a severe complication of Clostridium difficile infection in $1.36 \%$ of patients in the Clinical Infectious Diseases Hospital, Constanta. The risk factors for severe CDI evolution were: older age, obesity, significant leukocytosis, renal failure, marked hypoalbuminemia, malignancies, chemotherapy and ribotype 027.

Tigecycline was successful for the treatment of severe CDI, together with intravenous metronidazole and oral vancomycin. The beneficial effect of intracolonic administration of vancomycin as treatment in unresponsive CDI patients was noted in the three presented cases, resulting in a better outcome, and without the need of surgical procedures.

\section{ACKNOWLEDGEMENT}

This work has been supported by the grant EASME/ EMFF/2014/ 1.2.1.5/2/SI2. 707672 MSP LOT 1 BLACK SEA (MARSPLAN-BS), developed by Ovidius University of Constanța as partner, and financed by the European Commission.

\section{Author Disclosure Statement}

The authors report no conflicts of interest.

\section{DEFERENCES}

1. Cohen SH, Gerding DN, Johnson S, et al. Clinical Practice Guidelines for Clostridium difficile infection in adults: Update by the Society for Healthcare Epidemiology of America (SHEA) 
and the Infectious Diseases Society of America (IDSA). Infection Control and Hospital Epidemiology. 2010;31:431-455.

2. Sartelli M, Malangoni MA, Abu-Zidan FM, et al. WSES guidelines for management of Clostridium difficile infection in surgical patients. World J Emerg Surg. 2015;10:38.

3. Dallal RM, Harbrecht BG, Boujoukas AJ, et al. Fulminant Clostridium difficile: an underappreciated and increasing cause of death and complications. Ann Surg. 2002;235:363-72

4. Adams SD, Mercer DW. Fulminant Clostridium difficile colitis. Curr Opin Crit Care. 2007;13:450-5.

5. Rubin MS, Bodenstein LE, Kent KC. Severe Clostridium difficile colitis. Dis Colon Rectum. 1995;38(4):350.

6. Koo HL, Koo DC, Musher DM, DuPont HL. Antimotility Agents for the Treatment of Clostridium difficile diarrhea and colitis. Oxford Journals Medicine \& Health Clinical Infectious Diseases. 2009; 48(5):598-605.

7. Sayedy L, Kothari D, Richards RJ. Toxic megacolon associated Clostridium difficile colitis. World J Gastrointest Endoc. 2010;2(8):293-7.

8. Grigorescu BL, Fodor RS, Cioc AD, et al. Factors Favouring the Development of Clostridium Difficile Infection in Critically III Patients. The Journal of Critical Care Medicine. 2016;2(1):38-43.

9. Martin JS, Monaghan TM, Wilcox MH. Clostridium difficile infection: epidemiology, diagnosis and understanding transmission. Nat Rev Gastroenterol Hepatol. 2016;13(4):20616.

10. Sayedy L, Kothari D, Richards RJ. Toxic megacolon associated Clostridium difficile colitis. World J Gastrointest Endosc. 2010;2(8):293-297.

11. Stallmach A. Clostridium difficile infection: What is currently available for treatment? Internist (Berl). 2016;57(12):11821190.

10. Abou Chakra CN, McGeer A, Labbé AC, et al. Factors Associated With Complications of Clostridium difficile Infection in a Multicenter Prospective Cohort. Clin Infect Dis. 2015;61(12):1781-8.

11. Shivashankar R, Khanna S, Kammer PP, et al. Clinical factors associated with development of severe-complicated Clostridium difficile infection. Clin Gastroenterol Hepatol. 2013;11(11):1466-71.

12. Khanafer N, Barbut F, Eckert C, et al. Factors predictive of severe Clostridium difficile infection depend on the definition used. Anaerobe.2016;37:43-8.

13. Mulki R, Baumann AJ, Alnabelsi T, et al. Body mass index greater than 35 is associated with severe Clostridium difficile infection. Aliment Pharmacol Ther. 2016 Oct 28. doi: 10.1111/apt.13832. [Epub ahead of print]
14. Lu H, Wu Z, Xu W, Yang J, Chen Y, Li L. Intestinal microbiota was assessed in cirrhotic patients with hepatitis $B$ virus infection. Intestinal microbiota of HBV cirrhotic patients. Microb Ecol. 2011;61(3):693-703.

15. Aly AM, Adel A, El-Gendy AO, Essam TM, Aziz RK. Gut microbiome alterations in patients with stage 4 hepatitis C.Gut Pathog. 2016;8(1):42.

16. Naimushin A, Eliasaf S, Livneh A. Clostridium difficile-associated diarrhea: causes and relationship to reactive arthritis. Harefuah. 2011;150(1):64-6.

17. Yu JH, Kim NY, Lee HM, et al. A case of pseudomembranous colitis in a juvenile rheumatoid arthritis patient taking methotrexate. Korean J Gastroenterol. 2010;56(6):387-90.

18. Vaahtovuo J, Munukka E, Korkeamäki M, Luukkainen R, Toivanen P. Fecal microbiota in early rheumatoid arthritis. J Rheumatol. 2008;35(8):1500-5.

19. Ofosu A. Clostridium difficile infection: a review of current and emerging therapies. Ann Gastroenterol. 2016;29(2):147-54.

20. Arvand M, Hauri AM, Zaiss NH, Witte W, Bettge-Weller G. Clostridium difficile ribotypes 001, 017, and 027 are associated with lethal C. difficile infection in Hesse, Germany. Euro Surveill. 2009;14(45): pii: 19403.

21. Rao K, Micic D, Natarajan M, et al. Clostridium difficile ribotype 027: relationship to age, detectability of toxins A or B in stool with rapid testing, severe infection, and mortality. Clin Infect Dis. 2015;61(2):233-41.

22. Dubberke ER, Sadhu J, Gatti R, et al. Severity of Clostridium difficile-associated disease (CDAD) in allogeneic stem cell transplant recipients: evaluation of a CDAD severity grading system. Infect Control Hosp Epidemiol. 2007;28:208-11.

23. Henrich TJ, Krakower D, Bitton A, Yokoe DS. Clinical Risk Factors for Severe Clostridium difficile-associated Disease. Emerg Infect Dis. 2009;15(3):415-22.

24. Akamine CM, Ing MB, Jackson CS, Loo LK. The efficacy of intracolonic vancomycin for severe Clostridium difficile colitis: a case series. BMC Infect Dis. 2016;16:316.

25. Britt NS, Steed ME, Potter EM, Clough LA. Tigecycline for the Treatment of Severe and Severe Complicated Clostridium difficile Infection. Infect Dis Ther. 2014;3(2):321-331.

26. Gergely Szabo B, Kadar B, Szidonia Lenart K, et al. Use of intravenous tigecycline in patients with severe Clostridium difficile infection: a retrospective observational cohort study. Clin Microbiol Infect. 2016. doi: 10.1016/j.cmi.2016.08.017. [Epub ahead of print]

27. Larson KC, Belliveau PP, Spooner LM. Tigecycline for the treatment of severe Clostridium difficile infection. Ann Pharmacother. 2011;45(7-8):1005-10. 